among women with one or more previous negative smears. Women who are already in the screening system are probably more likely to have an invasive cancer diagnosed at an earlier stage than those who are not.

A further conclusion which may be drawn from this paper is that some $80 \%$ of patients developing invasive cancer did not have a previous negative screening test result, and hence must have slipped through the screening system because of failure to be screened at all, failure of adequate follow up, or failure of treatment. One case in the last category is quoted where a woman had been treated for intraepithelial neoplasia by cone biopsy and, despite six subsequent negative smears, eventually developed invasive cancer.

Though it is important to maintain high quality control at every stage of screening, the priority to be given to different measures to improve quality needs to be assessed in the light of the contribution of each to controlling the disease. Two recent studies, in Manchester and in south London, have looked at the reasons why women developing invasive cancer of the cervix had not been detected at a preinvasive stage by screening. ${ }^{67}$ By far the most common reason was that they had never been screened at all; this applied to two thirds of the total sample and four fifths of those over 40, among whom the great majority of cases occur. Failure to follow up abnormal cytological results accounted for about $15 \%$, with too infrequent screening - that is, an interval of over five years-and possible false negative results each contributing less than $10 \%$ of the total.

Hence in deciding priorities, recruitment of women into the screening system, particularly those past their childbearing years, still offers the best prospect of preventing a substantial number of women from developing invasive cancer. This is not to deny the increase in the incidence of the disease in younger women or to suggest that they should not be screened at all: rather to emphasise that screening resources should be more equitably shared among all those at risk. This is the essential message of the third paper in this week's issue, a statement by the ICRF Coordinating Committee on Cervical Screening ( $p$ 894). This outlines a plan for positive invitations to all adult women to be screened every five years, using a computerised invitation and recall scheme integrated with laboratory records, and incorporating a system that monitors that action has been taken on positive results. Such a scheme has been developed by the Exeter Family Practitioner Services Computer Unit, funded by the Department of Health and Social Security, and is now starting to be implemented in a few family practitioner committee areas. It has not yet been tested in practice and it remains to be seen whether exerting more positive control of the screening programme by a computerised system will make a sizable impact on the incidence of cervical cancer.

Meanwhile, as the fourth paper in this issue ( $p$ 883) shows, general practitioners can persuade virtually all the women in their practices to have smears. The $96 \%$ uptake rate reported by Standing and Mercer is attributable to their determination - culminating in home visits for women who did not respond to letters and telephone calls.

The principal reason for choosing a five yearly rescreening interval is cost. The clinician, whose priority is to do the best he can for his individual patient, may prefer to reconimend a shorter rescreening interval, as suggested by Paterson et al, but even one yearly screening will miss some fast growing cancers and endocervical adenocarcinomas. Health authorities, however, have a responsibility to the whole population they serve, and from this public health point of view a five yearly programme which reaches a high proportion of women and ensures appropriate action on those found positive will $\underline{\underline{T}}$ clearly prevent many more cases of invasive cancer than $\vec{s}$ frequently repeated smears on a small number of women. $\stackrel{\circledR}{\unrhd}$ Authorities may look at the priority to be given to prevention of cervical cancer (2000 deaths a year in England and Wales) alongside that of other preventive programmes for women, $D$ including lung cancer ( 8400 female deaths) and accidents $\mathbb{\infty}$ (8500 female deaths). In this context the provision of a m computerised system to implement and monitor five yearly screening of all adult women seems a very reasonable policy. $\overrightarrow{\vec{\omega}}$ Let us hope that it will result in a reduction in incidence of $\overrightarrow{0}$ invasive cervical cancer comparable with that achieved in other countries.

JOCELYN CHAMBERLAIN

Regional Specialist in Cancer Services,

South West Thames Regional Cancer Organisation,

Royal Marsden Hospital,

Sutton SM2 5PT

1 Hakama $M$. Trends in incidence of cervical cancer in the Nordic countries. In: Magnus K, ed. Trends in cancer incidence. Washington: Hemisphere, 1982:279-92.

Armstrong A Follow up of women with dysplasia of the uterine cervix. London: University of London, 1980. (PhD thesis.)

Kinlen LJ, Spriggs AI. Women with positive cervical smears but without surgical intervention. A follow up study. Lancet 1978;ii:463-5.

Department of Health and Social Security Health Services Development. Screening for cervical cancer. London: DHSS, 1984. (HC84(17),

Yule $\mathbf{R}$. The prevention of cancer of the cervix by cytological screening of the population. In: Easson EC, ed. Cancer of the uterine cervix. London: Saunders, 1984:11-25.

6 Chisholm DK, Haran D. Cases of invasive cervical cancer in the north west in spite of screening. British fournal of Family Planning 1984;10:3-8.

Prac R, Chamberlain J. Improving the effectiveness of cervical cancer screening. $7 \mathrm{R}$ Coll Gen Pract (in press).

\section{Children on motorcycles: a cause for concern}

Motorcycles are known to be dangerous machines, but in recent years much has been done to reduce the number of accidents, particularly among teenage riders, who account for almost half of all motorcycle casualties. ${ }^{1}$ Little or no attention has, however, been given to the injuries sustained by the ever growing numbers of children riding motorcycles for sport and pleasure. Sherman and Mackinnon's paper (p 877) presents some alarming data. Although their survey included only relatively few patients, it clearly showed the potential for serious injury for both the supervised and unsupervised riders. They rightly emphasise the need for $ᄋ$ further study: their experiences are unlikely to be unique, and probably cases of the kind they describe are occurring throughout Britain.

By law children aged under 16 are forbidden to ride a $>$ powered two wheeled vehicle on the public highway, but that does not prevent their riding motorcycles on private pro- $N$ perty. Since the Autocycle Union established its youth division in 1974, clubs catering for motorcycle riders aged $\bar{\sigma}$ between 6 and 17 have proliferated. The rules governing the conduct of the members of these clubs are comprehensive $\Phi$ and ensure that children ride with maximum safety. Many $\stackrel{+}{+}$ more children, however, ride unsupervised in fields and on waste and common ground. In these circumstances the pro- $\frac{\overrightarrow{\mathbb{D}}}{\mathrm{D}}$ vision of a safe motorcycle, adequate training, and protective $\frac{\vec{\Phi}}{\mathbb{D}}$ clothing becomes the sole responsibility of the parents or guardians.

We have few data on the injuries sustained during the $\frac{8}{0}$ pursuit of this activity; the details that are available relate only to motorcycle accidents occurring on the public highway, and even then non-fatal accidents are underreported. ${ }^{2}$ 
Nevertheless, we can expect that by virtue of their immature coordination and judgment the accident rate in children will be particularly high, especially among the unsupervised riders. Since these motorcycles are being ridden off the road accidents are less likely to involve a second vehicle-indeed, about one third of motorcycle accidents on public roads result from riders falling off their machines, but are no less damaging for that."

Can it be wise to permit children as young as 6 to ride machines of $50 \mathrm{cc}$ and over at speeds of up to 40 miles an hour? Rather than permit the unrestricted sale of motorcycles designed for children, would it not be more responsible to restrict their sale to recognised clubs for use at official meetings only and to raise the lower age limit? Should we not ensure that a medical officer is present at all such meetings and that accidents causing injury are fully documented in order that safety measures in force may be evaluated?

Motorcycling is potentially a most dangerous sport and is probably not appropriate for children under the age of 12: unsupervised, the riding of motorbikes by children of any age is quite unacceptable and should be outlawed.

M A MASON

Senior Registrar,

Accident and Emergency Department,

Northampton General Hospital,

Northampton NN1 8BD

1 Royal Society for the Prevention of Accidents. 1982 road accident statistics. London: Royal Society for the Prevention of Accidents, 1984:4.

Woodward A. Motorcycle accidents in Nottinghamshire. Public Health 1984;97:139-48. Transport and Road Research Laboratory. Injuries to unprotected road users. Crowthorn: Transpor
and Road Research Laboratory, 1979. (Leaflet No LF 792.)

\section{Replacement of surfactant in hyaline membrane disease}

\begin{abstract}
If hyaline membrane disease is due entirely to surfactant deficiency, as is widely believed, it should be amenable to replacement treatment. Indeed, attempts at replacement of surfactant have been made for 20 years, ${ }^{12}$ but encouraging results have been obtained only recently. The practical problems may be summarised in four questions: What should be given as replacement? How much should be given? How should it be administered? and When is it best given?
\end{abstract}

The first question begs another: what is pulmonary surfactant? Natural surfactant lipoprotein is made up roughly of $45 \%$ dipalmitoyl phosphatidyl choline, $20 \%$ phosphatidyl choline containing unsaturated fatty acids, $6 \%$ phosphatidyl glycerol, $5 \%$ protein, $12 \%$ other phospholipids, and $9 \%$ neutral lipids. ${ }^{34}$ But an adequate answer to the question requires further detail of the physical states through which natural surfactant passes in its cycle of secretion and reabsorption. ${ }^{4}$ Surfactant is manufactured by alveolar type II cells and is stored intracellularly in osmophilic lamellar bodies. ${ }^{4}$ These bodies are secreted into the thin layer of liquid which coats the alveoli, where they unravel and recombine to form tubular myelin. ${ }^{5}$ The unique structure of tubular myelin (long lipid walled tubes of square cross section which are closely packed together) is seen only in natural surfactants containing physiological concentrations of calcium. ${ }^{\circ}$ The specific surfactant apolipoprotein may be necessary for the structure of tubular myelin, a notion which is supported by the fact that dipalmitoyl phosphatidyl choline binds the apolipoprotein preferentially.

The extremely rapid adsorption of natural surfactant to an air-liquid surface is due, probably, to some of the lipids being in the form of tubular myelin. ${ }^{6}$ The structure may be nature's way of prepackaging the hydrophobic "tails" and hydrophilic polar "heads" of the phospholipids in large continuous sheets (the walls of the tubes) so that the lipid can adsorb to a surface with less energy and thus faster than if the lipids were in the form of liposomes. ${ }^{8}$ After a period of "aging" the lipids desorb from the surface monolayer and form liposomes or vesicles, which are recycled by the type II alveolar cell or ingested by alveolar macrophages. ${ }^{9}$ (By no means are all of these concepts universally held: some think that tubular myelin is an inactivated form of surfactant ${ }^{10}$; others that the surfactant monolayer is not applied on an aqueous hypophase but directly on to the epithelial cells of the lung. ${ }^{11}$ )

To return, then, to the first question: What is the best surfactant to use clinically? The answer must take account not only of the constituents but also of the adsorption rate of the mixture and its ability to give consistently very low surface tensions on repeated dynamic compression of a surface monolayer in vitro; perhaps even a note on its ultrastructure should be included. If the scheme of events described above is correct then tubular myelin, the immediate precursor of the surface monolayer which is found only in natural surfactants, would be the "best" surfactant to use. Natural surfactants, however, contain protein (plasma proteins as well as the apolipoprotein, all of which might be antigenic), so paediatricians have been understandably reluctant to use animal surfactants in babies, preferring synthetic or artificial mixtures containing only lipids.

Dipalmitoyl phosphatidyl choline, the main constituent of surfactant, is a solid at temperatures up to $41^{\circ} \mathrm{C}$ and adsorbs hardly at all to an air-liquid surface. The addition of other lipids, particularly phosphatidyl glycerol and some containing unsaturated fatty acid side chains, may lower the temperature at which the dipalmitoyl phosphatidyl choline will adsorb and spread on a surface.12 "Dry" (solid) surfactant, a mixture of dipalmitoyl phosphatidyl choline and phosphatidyl glycerol in powder form, circumvents the adsorption hurdle by spreading extremely rapidly (in vitro), provided that it is delivered from the air side of an air-liquid surface. ${ }^{8}$ Adsorption of dipalmitoyl phosphatidyl choline from the aqueous phase may also be enhanced by the addition of plasma high density lipoprotein ${ }^{12}$ and surfactant apolipoprotein, ${ }^{72}$ though this action has been disputed. ${ }^{13}$ Unfortunately, even at best, the adsorption from an aqueous phase of synthetic surfactants, ${ }^{12}$ and even of lipids extracted from natural surfactants and therefore containing hardly any protein, ${ }^{14}$ is two orders of magnitude slower than of natural surfactant containing tubular myelin, which adsorbs within seconds. ${ }^{6}$

These facts help to explain why the results of clinical trials of synthetic surfactants have been disappointing. ${ }^{12} 15$ Nevertheless, Morley et al claimed that babies treated with "dry" surfactant fared better than an untreated group ${ }^{16}$; but the trial was poorly controlled, and two other small clinical studies of the same dry surfactant showed no effect. ${ }^{17} 18$ Morley and his coworkers have abandoned surfactant in powder form and are currently assessing the effect of a cold aqueous slurry of "dry" surfactant. A different synthetic 